\title{
The Research Environment of Universities
}

\section{Javier Vidal and Miguel Quintanilla}

The aim of this study is to describe the real environment of a university with reference to its research activities and to measure the influence of geographical proximity as a decisive factor in the establishment of collaboration between the university and other institutions. To do this analysis, a case study has been carried out on two Spanish Universities: the University of Leon and the University of Salamanca. The main conclusion is that the environment of a university does not necessarily consist of elements from its closest geographical environment. We have also identified some features of university-company relations that suggest that geographical proximity does not guarantee greater opportunities for relationships. The two most important factors seem to be the companies' attitude towards innovation and the personal contacts of researchers.

\section{Introduction}

On many occasions, when we talk about Highe Education Institutions we refer to their environment. For instance, one of the objectives for these institutions is usually to promote or to collaborate in the development of the economy and culture of this environment (see Mora 1991 for objectives and benefits of higher education). This is also one of the main arguments for the creatio of a new university in a specific location, especially if it is a public university. But exactly what do we mean by 'their environment'? Probably, the most common meaning is 'the neighbouring geographical environment', and this is exactly what we have in mind when we use this argument to support the creation of new universities.

On the other hand, one can view a university from the prespective of systems theory. From this point of view, universities can be seen as open and complex systems (Höltta and Pulliainen 1996). These two characteristics are connected with our problem. The environment depends on how open the system is and the relationships among the components of the system, including the environment, determine how complex it is. So, we have practical and theoretical reasons identifying the extent to which geographical proximity is the most important factor when attempting to define this element of the system.

From now on we shall only talk about one of the dimensions of universities - that of research. So, when we say environment, we refer to the research environment, meaning all the institutions, public or private, that have any kind of relation with the research activities of a university. These relations can be research collaboration (scientific collaboration), research contracts and funding. We considered the importance of all the aspects related to teaching for the problem we are presenting, but concluded that taking these two dimensions together would be very complex. We

Dr.Javier Vidal is Director of the Quality Institutional Programme at the University of Leon. Edificio Biblioteca Universitaria. E-24071 Leon, Spain. Tel.: + 34987291052 . Fax: + 34987 291622. E-mail: dfcjug@unileon.es 
decided, therefore, to focus our attention on only the research dimension.

In many discussions about the environment of a university there is a debate between what we think a university should be and what it in fact is. In this paper we shall present some information about what such an environment really is and draw some policy implications. Connecting research with reality, the problem of the environment of universities may be expressed briefly as who wants to do what and pay for it? Before going on with the analysis, let us provide a few details to understand this situation in the Spanish context.

First, the Spanish University System has grown quickly. Since 1975, 26 public universities have been set up. That is almost half of all public universities in the country. These new universities are located across the whole of Spanish territory and it is clear that there has been a regional distribution of these new universities. There have also been some reforms since 1983 that have affected the university system. The most important of these has been the University Reform Act and the Science and Technology Act (for more information about the development of the Spanish university system, see Sánchez Ferrer 1996; Mora 1991, 1996, 1997).

Second, most Spanish universities are public and a process has just been completed whereby the control of funds for universities has moved from the hands of the national government to the regional governments. In general, these regional governments seem to be more interested in making the universities useful within their own regions, rather than in prompting them to spend their time and money on national or transnational relations. In other words, there is a conflict between regional scientific and technological interests and national science and technology policy that is sometimes very difficult to confront. There is also another more general contradiction related to this: effective responses to the external demands of education and research services may be considered as a threat to academic values and institutional autonomy (Höltta and Pulliainen 1996). So there are two aspects: policy in general versus academic autonomy, and national policy versus regional policy.

Third, the problem of internal changes (institutional changes) is specially stressed because nearly all researchers have sufficient autonomy for example, to contact companies. It is, therefore, very difficult to design and implement an institutionally co-ordinated research policy.

Fourth, research activities at universities are very important not only for the university itself but also for the Spanish Science, Technology and Industry System - 50 per cent of researchers in Spain are in universities and they spend one-fourth of total $R \& D$ expenditures. These figures are very high but, nonetheless, we should take into account the fact that many researchers a universities work in the field of the Humanities and have almost no links at all with $\mathrm{R} \& \mathrm{D}$ activities. However, Spanish science has a very prominent academic character and the relations between university and industry have grown, probably as a consequence of the national science policy of the last decade.

Summing up, we have a public-based university system which has expanded enormously over the past two decades, has undergone important reforms, is now in the hands of the regional governments, has a considerable degree of autonomy for institutions and researchers, and is very important in terms of the National System of Science, Technology and Industry.

\section{Objectives and methodology}

With this context in mind, we have defined two objectives: first, to describe the real environment of a university institution as regards the research activities carried out within it and second, to measure the influence of geographical meximity a decisive factor in the establishment of collabration between the university and other instituions for the purposes of research.

To do this analysis, a case study has been carried out on two Spanish universities - the University of Leon and the University of Salamanca. These two universities belong to the same region, Castilla y Leon, but have entirely different characteristics. The University of Leon is a small new university $(14,000$ students in 1993-94) that was created in 1979. It is strongly oriented towards the veterinary, agricultural and biological sciences. The University of Salamanca is the old est university in Spain (and, indeed, one of the oldest in Europe). It is a medium-sized institution (30,000 students in 1993-94) and can be said to be strongly oriented towards the Health Sciences and Humanities.

To perform this analysis we selected a dua methodology in order to gain a quantitative description of research collaboration and a qualitative explanation of why this type of collaboration, and not other types, emerges. On the one hand, we selected some quantitative indicators based on research projects, research contracts and scientific publications compiled in the Science Citation Index (SCI). From the latter, we only analysed the documents written in collaboration. Owing to the availability of information, we chose different periods, from 1988 to 1995 For all of these factors (projects, contracts and publications), we determined four possible geographical levels according to where each institution or company that had had any relationship with the university was based. These levels are the local, regional, national and transnational

Table 1: Sources of research funds, University of Leon, 1988-92

\begin{tabular}{|l|c|c|c|c|}
\hline \multicolumn{5}{|c|}{ Geographical Level } \\
\hline Type of institution & Local & Regional & National & Transnational \\
\hline Public institutions & $13 \%$ & $24 \%$ & $56 \%$ & $8 \%$ \\
\hline Enterprises & $35 \%$ & & $33 \%$ & $32 \%$ \\
\hline Total & $17 \%$ & $19 \%$ & $51 \%$ & $12 \%$ \\
\hline
\end{tabular}

Table 2: Sources of research funds, University of Salamanca, 1990-94

Geographical Level

\begin{tabular}{|l|c|c|c|c|}
\hline Type of institution & Local & Regional & National & Transnational \\
\hline Public institutions & $35 \%$ & $32 \%$ & $33 \%$ & $*$ \\
\hline Enterprises & $3 \%$ & & $48 \%$ & $49 \%$ \\
\hline Total & $12 \%$ & $9 \%$ & $44 \%$ & $35 \%$ \\
\hline
\end{tabular}

* There is an error in the database and there is no precise information about transnational relations with public institutions, although we know that there are some. Nevertheless, the real situation should be then more favourable than assumed in our conclusions.
We selected this type of information ronment that has been given.

One other hand, we interviewed 36 reResults

Tesuls of the quantitative analysis are shown to 4 . As can be seen, the national and this versities and both groups of indicators - from 63 列 one can be found in local level collaboration with the collaboration exerthe University of Leon with an impor列 town. By contrast, the University of Salamanca has more publications in collaboration with local public institutions because there is an important 3. Despite 79 per cent in Tables 2 and 
Table 3: Collaboration with other institutions, Publications (SCI), University of Leon, 1988-92

\begin{tabular}{|l|c|c|c|c|}
\hline \multicolumn{5}{|c|}{ Geographical Level } \\
\hline \multicolumn{1}{|c|}{ Type of institution } & Local & Regional & National & Transnational \\
\hline Universities & & $18 \%$ & $62 \%$ & $20 \%$ \\
\hline Public Research Centres & $32 \%$ & $4 \%$ & $65 \%$ & \\
\hline Enterprises & $26 \%$ & $19 \%$ & $4 \%$ & $50 \%$ \\
\hline Other & & $5 \%$ & $48 \%$ & $48 \%$ \\
\hline Total & $4 \%$ & $16 \%$ & $56 \%$ & $23 \%$ \\
\hline
\end{tabular}

Table 4: Collaboration with other institutions, Publications (SCI), University of Salamanca, 1990-95

\begin{tabular}{|c|c|c|c|c|}
\hline \multicolumn{5}{|c|}{ Geographical Level } \\
\hline Type of institution & Local & Regional & National & Transnational \\
\hline Universities & & $5 \%$ & $43 \%$ & $52 \%$ \\
\hline Public Research Centres & $54 \%$ & & $21 \%$ & $25 \%$ \\
\hline Enterprises & $3 \%$ & $3 \%$ & $89 \%$ & $5 \%$ \\
\hline Other & $41 \%$ & $8 \%$ & $41 \%$ & $10 \%$ \\
\hline Total & $25 \%$ & $4 \%$ & $35 \%$ & $36 \%$ \\
\hline
\end{tabular}

centre of the Higher Council for Scientific Research in Salamanca. These differences are not relevant to our analysis because they refer to the type of institution and not their geographical level. Additionally, such differences are to be expected in case study methodology.

In general, the external relations that the Universities of Leon and Salamanca have concerning research issues can be said to lie basically within the national and transnational environment. Relations with companies also lie at the national and transnational level. It should be noted that there is an absence of relations with companies from the regional environment and that relations with transnational companies are scarce and highly concentrated in certain specific scientific fields Thus geographical proximity does not seem to be the definitive factor.

see whether this situation is peculiar to this region (Castilla y Leon), Figure 1 represents, by regions, the total number of SCI documents, the total number of businesses and the population of the area. The map indicates where the population and companies are located and where the regions with the greatest scientific production are. These are Madrid and Catalonia, and, at a lower level, Valencia and Andalusia. What this shows is that the population, the number of companies and scientific production are very concentrated. Accordingly, for the rest of the regions, it is easier to collaborate with institutions from these more important regions than with institutions from their For instance, the University of Leon collaborates more with Madrid than with any other part of its own region. This is further evidence that geographical proximity is not a definitive factor. In this case, the number of companies

Figure 1

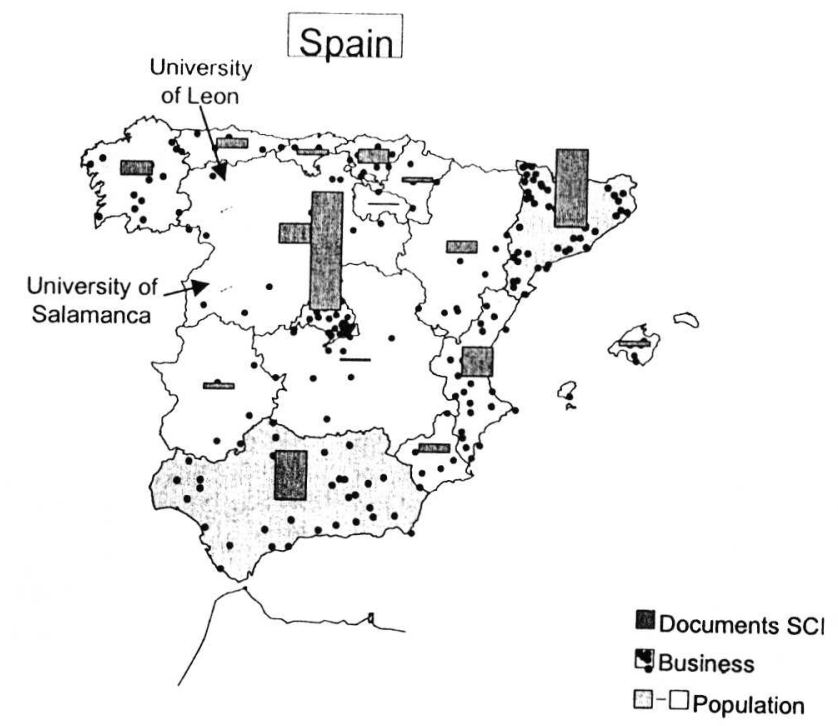

Sources: INE (http://www.ine.es/htdocs/espa96/espcif96.htm) Maltrás and Quintanilla (1995)

Periods: Documents SCI: 1986-91. Business: 1996. Populations: 1991.

and total scientific production (as an indicator of scientific activity) seem to be more decisive in explaining scientific collaboration.

This situation was analysed in the interviews, with the same results. As we have said, we attempted to determine which factors affect the setting up of relationships with other research institutions or companies. The two most important conclusions obtained through these interviews are as follows (see similar conclusions in Höltta and Pulliainen 1996, p. 123).

First, relationships with the neighbouring geographical environment depend, partly on the type of research. For instance, research focused on local issues (e.g. agriculture) reduces the possibilities of establishing relationships with other institutions at a higher geographical level ('our research has been of local interest and therefore has no international impact'). On the other hand, companies from neighbouring geographical environments cannot absorb the research carried out in certain other scientific fields (e.g. biotechology)

Second, contacts with other centres - public or private - are mainly generated through personal relationships among researchers and not so much through actions promoted by the institutions themselves. These personal relationships are not affected by the proximity of institutions but ather by social factors such as the place where the researcher has studied ('when I was in USA, I made many contacts with companies') or worked ('we have many contracts with this company because this new professor used to work there', 'I still have contacts with my previous university') The personal relationship factor was the one most cited as the key to understanding the relationships of researchers with 'the outside world'.

In principle, these two elements suggest that geographical proximity is not the decisive factor explaining these types of collaboration relationships. As Skilbeck (1997) points out, it is not only a problem of commitment but also of the capacity 
of public and private institutions to establish relations with universities

Thus the two types of information that we have used lead us to the same conclusion. However, before going deeper into this general conclusion, let us now move on to some other interesting details. According to the various opinions received there are many nuances and different explanations to account for the main features that characterize external relations with companies.

Each scientific discipline has a different attitude towards companies. Some researchers consider that it is impossible to establish contacts with companies because of the very nature of their types of research, especially in the field of the Humanities. It should be recalled that there are many researchers in the Humanities at Spanish universities. Others, however, consider this type of relationship of great importance for their work. Owing to different opportunities, and probably also to different experiences, there is a plethora of points of view in this respect. Let us look at some results related to the type of companies with which researchers can contract research.

First, contacts with companies are set up through personal relationships between researchers from both types of institutions. Sometimes the companies take the initiative, while on other occasions it is the universities who do so. Cases of both strategies have been identified, both with good results. Therefore, in principle, neither approach seems to be better than the other. This implies that it is sometimes difficult to set up common strategies to promote university-company relationships and to co-ordinate these actions since, for instance, researchers are reluctant to offer their personal contacts to the central services of the university. Indeed, in certain respects they are very possessive about them. These personal contacts may arise in many ways - study visits, congresses, etc. Accordingly, it does not appear that the closest geographical environment will provide greater opportunities for $\mathrm{col}-$ raration.

Second, there are many reasons why companies maintain relationships with universities: (a) they seek solutions, often rapid solutions, to specific problems:

(b) they wish to bring their staff up to date;

(c) they look for cheap information and projects (the most frequent opinion is that 'companies give little and demand a lot');

(d) they look for academic guarantee or the prestige of a research group for their projects, mainly when they are looking for public funding;

(e) there are also some companies that are interested in evaluation studies (processes, products or the company itself) to be implemented from outside, in a search of impartiality; and

(f) occasionally, companies have other practical reasons for setting up contracts with universities. For example, it is sometimes cheaper to experiment in institutions in other countries - sometimes this is less risky or 'it is cheaper to contract outside than to build a laboratory'.

All this means that companies look for what they necessarily in the nearest universities or institutions.

Third, it seems that there are as many specific problems in companies, as in universities, regarding the establishment of new relationships with each other. It is a recognized fact that the companies closest to the University of Leon demand little research from it, in spite of the fact that the most important scientific fields of expertise of the University of Leon (veterinary and agricultural sciences) are very germane to the local economy. A possible explanation for this might be a certain lack of innovative culture in the companies, their small size (COTEC 1994) and the conviction on the part of company managers that the university is unable to provide solutions, often rapid solutions, to their problems. Another likely reason is that researchers are sometimes unaware of what can be done in joint activities with companies. Fourth, some of the contracts that apparently come from companies are, in fact, financed by public funds. In this sense, the initiative in these cases almost invariably comes from the national science policy.

Conclusions and policy implications

What has been said so far leads us to conclude What he research environment of a university is that the rescarch envile up elements from the not necessarily made up of lements from the nearest geographical environment. On the one hand, a description has been given of the relationships set up over a five-year period for two Spanish universities. It has been demonstrated that the national and transnational research envithat the nas more weight in all the variables ana lyzed than the local and regional environment. On the other hand, some features that characterize university-company relations have been detected that suggest that geographical proximity does not guarantee greater opportunities for reladoes nips, even when both parties work in simila tionships, even whon both fields. The two most important factors seem to be the attitude of companies themselves towards in novation and the personal contacts of researchers.

To sum up, these universities do not have many relations with their nearest companies, whereas they do have important relationships with national and transnational companies and institutions. This suggests that there are other kinds of environments that can be more suitable for policy planning The environment that we propose might be called a disciplinary environment and this is defined as the total number of institution and companies that can collaborate with a university depending on the scientific specialization and quality of the university research groups. Of course, this does not exclude companies and institutions from the nearest geographical environment but it does mean that disciplinary proximity is a more decisive factor than geographical proximity.

In spite of all of this, we still have to answer the question of whether it is possible for a university to promote the creation of new companies in its neighbouring geographical environment. One may assume that this is possible, although, according to our results, it is not a sufficient condition. Companies do not need to be near a university to set up relationships with it. In fact, in Spain, companies - which are mainly located in Madrid and Barcelona - do not find it difficult to contact universities from all over Spain and even abroad. So, although it may be true that a university can be a tool for regional development policy, this is probably so for reasons other than the influence of its research activities on its neighbouring environment. Analysis of these other reasons will be the topic of a future paper.

\section{Not}

The authors offer special thanks to Bruno Maltras and Sonia Martin of the University of Salamanca for the information provided for this paper.

\section{References}

COTEC (1994) Entorno y Tecnologia. Madrid: Fundación COTEC para la Innovación Tecnológica.

Höltta, S. and Pulliainen, K. (1996) 'The Changing Regional Role of Universities'. Tertiary Education and Management 2 2, 119-126.

Matrás, B. and Quintanilla, M.A. (1995) Spain Indicators on Scientific Production, 1986-1991. Madrid: CSIC.

Mora, J.-G. (1991) Calidad y rendimiento en las instituciones universitarias. Madrid: Consejo de Universidades. Mora, J.-G. (1996) 'The Demand for Higher Education in Spain'. European Journal of Higher Education 31, 3, $341-354$

Mora, J.-G. (1997) 'Market trends in Spanish higher education'. Higher Education Policy 10, 3/4, 187-198. Sánchez Ferrer, L. (1996) Políticas de reforma universitaric en España: 1983-1993. Madrid: Instituto Juan March de Estudios e Investigaciones.

Skilbeck, M. (1997) 'Higher Education in a Changing Environment: Regional, National and Trans-Nationa Issues'. Tertiary Education and Management 3, 2,

$101-111$ 